\title{
Editorial
}

\section{Ion-Exchange Membranes}

\author{
Yoshinobu Tanaka, ${ }^{1}$ Seung-Hyeon Moon, ${ }^{2}$ Victor V. Nikonenko, ${ }^{3}$ and Tongwen $X u^{4}$ \\ ${ }^{1}$ IEM Research, 1-46-3 Kamiya, Ushiki-shi, Ibaraki 3001216, Japan \\ ${ }^{2}$ Department of Environmental Science and Engineering, Gwangju Institute of Science and Technology (GIST), \\ 261 Cheomdan-Gwagiro, Buk-gu, Gwangju 500-712, Republic of Korea \\ ${ }^{3}$ Membrane Institute, Kuban State University, Stavropolskaya Street 149, Krasnodar 350040, Russia \\ ${ }^{4}$ CAS Key Laboratory of Soft Matter Chemistry, Laboratory of Functional Membranes, School of Chemistry and Material Science, \\ University of Science and Technology of China, Jinzai Road 96, Hefei, Anhui 230026, China
}

Correspondence should be addressed to Yoshinobu Tanaka, fwis1202@mb.infoweb.ne.jp

Received 7 August 2012; Accepted 7 August 2012

Copyright (c) 2012 Yoshinobu Tanaka et al. This is an open access article distributed under the Creative Commons Attribution License, which permits unrestricted use, distribution, and reproduction in any medium, provided the original work is properly cited.

Principles of ion transport across ion-exchange membranes had been investigated using biological membranes in old time. Industrial application of the membranes started after the invention of artificial membranes in 1950. Ion-exchange membrane electrodialysis is now one of the basic technology in saline water desalination industry. It is also applied to many fields such as demineralization and reuse of sewage or industrial waste, refining of amino acid solutions, production of organic and inorganic chemicals, membrane reactors, among others. Further, the membranes are applied to the succeeding technology such as electrodialysis reversal, bipolar membrane electrodialysis, electrodeionization, electrolysis, diffusion dialysis, fuel cell, reverse electrodialysis, among others.

This special issue presents the newest investigation on ion-exchange membranes submitted from each field. It includes topics in the fundamental studies and application studies such as membrane preparation, membrane characterization, membrane application, environmental problem; process design and operation, among others. Fundamental studies are the basis of the application studies. At the same time, the application studies induce the development of the fundamental studies. Both studies influence each other and consequently contribute to the technology development. Based on the history of ion-exchange membranes, the following ten articles published in this special issue must induce the development of succeeding technology.

In the first article "Reactivity of phenol allylation using phase-transfer catalysis in ion-exchange membrane reactor," $\mathrm{Wu}$ and $\mathrm{Fu}$ have reported the reactivity of phenol allylation using quaternary ammonium salt phase-transfer catalysts in ion-exchange membrane reactors incorporated with the laboratory-produced membrane. They have immobilized the catalyst in the pore of an ion-exchange membrane. That allowed them to benefit the permselective as well as the catalytic functions of the new membrane. The study investigates the reactivity of phenol allylation using quaternary ammonium salt as a phase-transfer catalyst in several types of membrane reactors. Optimum reactivity and turnover of phenol allylation were obtained using a respond surface methodology. The contact angle, water content, and crosslinkage degree were measured to understand the microenvironment in the ion-exchange membrane.

The second article is "Performance of a $1 \mathrm{~kW}$ class nafionPTFE composite membrane fuel cell stack" addressed by Kirshnamurthy et al. The membranes have been prepared by impregnation of Nafion into the expanded polytetrafluoroethylene (EPTFE) matrix. Nafion loading in the membranes was kept at lower amount of $2 \mathrm{mg} / \mathrm{cm}^{2}$. The lower amount of electrolyte per unit area in the composite membranes offers cost advantageous compared to conventional membranes. The composite membranes ( $30 \mu \mathrm{m}$ thickness) had higher thermal stability and mechanical strength compared to the conventional membranes $(50 \mu \mathrm{m}$ thickness). The durability of single pass cells was tested. The performance of the membrane electrode assembly (20-cell stack, $330 \mathrm{~cm}^{2}$ active area) have been reported and it was comparable to that of the conventional membrane.

In the third article, "Study of chromium removal by electrodialysis of tannery and metal-finishing effluents" by Moura 
et al., the membranes were synthesized from blends of polystyrene and polyaniline. The electrodialysis experiments were performed using a three-compartment cell with a capacity of $200 \mathrm{~mL}$ each. The unit was incorporated with the synthesized cation-exchange membranes (and Nafion 450) and anion-exchange membranes Selemion AMT. Supplying metal-finishing effluents collected at two industries, chromium removal percentage was evaluated. The synthesized membranes presented similar chromium transport to that observed in the Nafion 450. The study proved the feasibility of a technology in the treatment of tannery and metal finishing effluents, bringing great advantageous to water reuse.

In the forth article "Ion-exchange membranes based on polynorbornenes with fluorinated imide side chain groups", Santiago et al. have prepared cation-exchange membranes based on polynorbornenes with fluorinated and sulfonated dicarboximide side chain groups. The study was extended to block copolymer containing structural units with phenyl and 4-oxybenzenesulfonic acid, 2,3,5,6-tetrafluorophenyl moities replacing the hydrogen atom of the dicarboximide group. They discuss electrochemical characteristics of the membranes, electromotive forces of concentration cells, proton conductivity, and also proton permselectivity. The efficient segregation of hydrophilic from hydrophobic moieties, presumably favored by the low polarity of bonds attached to the phenyl groups, gives raise to the formation of percolation paths responsible for the rather high proton conductivity of the homopolymeric membranes.

The fifth article is "Measurement of membrane characteristics using phenomenological equation and the overall mass transport equation in ion-exchange membrane electrodialysis of saline water" by Tanaka. In saline water electrodialysis, the author found that the overall solute permeability $\mu$ occasionally takes minus value. For understanding this phenomenon, new concept of the overall concentration reflection coefficient $\sigma^{*}$ was introduced. $\sigma^{*}$ was defined for describing the permselectivity between solutes and water molecules (solvent) in the electrodialysis system just after an electric current interruption. Negative $\mu\left(\sigma^{*}<1\right)$ means that ions are transferred with water molecules from desalting cells toward concentrating cells just after an electric current interruption, indicating up-hill transport or coupled transport between water molecules and solutes.

The sixth article by Ayala-Bribiesca et al. "Ultrathin sicopion composite cation-exchange membrane: characteristics and electrodialytic performance following a conditioning procedure" has addressed the preparation of ultrathin $(\fallingdotseq 20 \mu \mathrm{m})$ highly conductive composite cation-exchange membranes. The membranes were made from sulphonated poly(etherether-ketone) containing different levels of sulphonic functionalized silica particles (SFSPs). Sicopion membranes were conditioned according to the French normalizing association procedure, and their electrodialytic characteristics were compared to an existent commercial food-grade membrane (CMX-SB). Electrical conductivity of Sicopion membranes was higher than that of CMX-SB membranes as well as their water content. As the SFSP level was reduced, the ionexchange capacity increased. Sicopion membranes presented a lower demineralization rate than CMX-SB membranes due to an $\mathrm{OH}^{-}$leakage through the pores created by dislodging the SFSP particles during the conditioning procedure.

The seventh article is "Hybrid anion exchange hollow fiber membrane for delivery of ionic drugs". Wang et al. prepared anion-exchange hollow fiber membranes based on bromomethylated poly(2,6-dimethyl-1,4-phenylene oxide) as drug carriers for some anionic model drugs such as sodium salts of benzoate, salicylate, among others. The above organic-inorganic hybrid anion exchange hollow fiber membrane was used for controlled release of the model drugs. They have established that the adsorption/release behavior of the membrane depends on the drug nature. In particular, the physicochemical characteristics of drugs are crucial to the interaction patterns between the drugs and the membrane, including electrostatic interactions and nonelectrostatic interactions (hydrophobic interactions and hydrogen bonding). The hydrogen bonding capacity of the drugs significantly affects the loading capacity and the release rate.

In the eighth article "Obtaining the zwitterionic Form of L-Lysine from L-Lysine monohydrochloride by electrodialysis" by Aghajanyan et al., L-lysine monohydrochloride was transformed to its zwitterionic form in four- and two-chamber electrodialysis apparatus. The process of transformation at various concentration of L-lysine monohydrochloride (0.1$0.7 \mathrm{~mol} / \mathrm{l})$ was studied. It was established that at the optimum current density in the chosen range of salt concentration, total transformation into its zwitterionic form occurred. In the process of transformation, changes in $\mathrm{Cl}^{-}$ion concentration, $\mathrm{pH}$, dry matter content, electroconductivity, as well as in current voltage depending on time were determined. Studies have shown that the process of total transformation was accomplished when $\mathrm{pH}$ of the lysine solution achieved 10. The losses of lysine diffused into the next chamber was less than $1.0 \%$. The specific energy consumption in two- and four-chamber electrodialyzer was 1.85 and $3.82 \mathrm{kWh} / \mathrm{kg}$ and the current efficiency was $\sim 76$ and $\sim 73 \%$ correspondingly.

In the ninth article "Enhancing ion transfer in overlimiting electrodialysis of dilute solutions by modifying the surface of heterogeneous ion exchange membranes", Pismenskaya et al. have studied the effect of surface modification of heterogeneous ion-exchange membranes on the ion transfer rate in overlimiting electrodialysis of dilute solutions. One of the membranes was obtained by casting a thin film of a Nafiontype material on the surface of a heterogeneous cationexchange membeane. The other membrane was made by grafting quaternary ammonium bases onto the surface layer of an anion-exchange membrane to replace secondary and tertiary ammonium groups initially present. The surface modification resulted in a considerable increase, up to two times, of mass transfer rate due to enhanced electroconvection at the cation-exchange membrane and suppressed water splitting at the anion-exchange membrane.

In the tenth article, "Ion transport through diffusion layer controlled by charge mosaic membrane," Yamauchi has studied charged mosaic membranes imposed on a cationexchange membrane or on an anion-exchange membrane. The author found that these complex two-layer membranes show properties typical for monopolar (selective transfer at 
the interface of monomer and mosaic layers) as well as for bipolar membranes (water splitting at the interface of monomer and mosaic layers). It was also established that the transition time in chronopotentiometric measurements was function of what monopolar membrane was used together with the mosaic one: in the case of the cation-exchange membrane, the transition time was higher, and in the case of the anion-exchange membrane, lower than that found for relative monopolar membranes. The thickness of the boundary layer was derived with conjugation with the limiting current density and the transition time.

In publishing the special issue, the editors thank deeply the authors for their excellent contributions and reviewers for their time and effort in reviewing the manuscripts.

Yoshinobu Tanaka
Seung-Hyon Moon
Victor V. Nikonenko
Tongwen Xu 

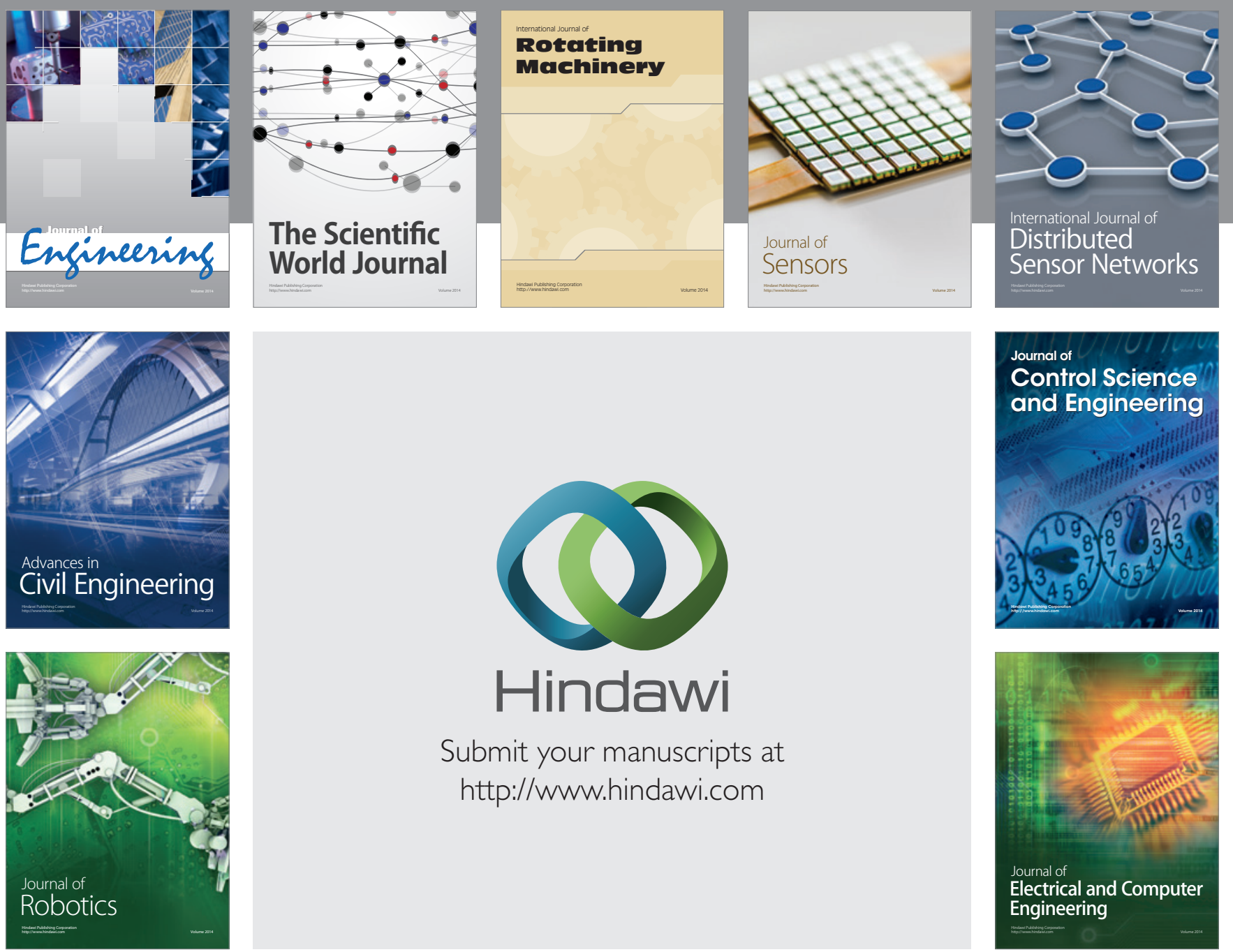

Submit your manuscripts at

http://www.hindawi.com
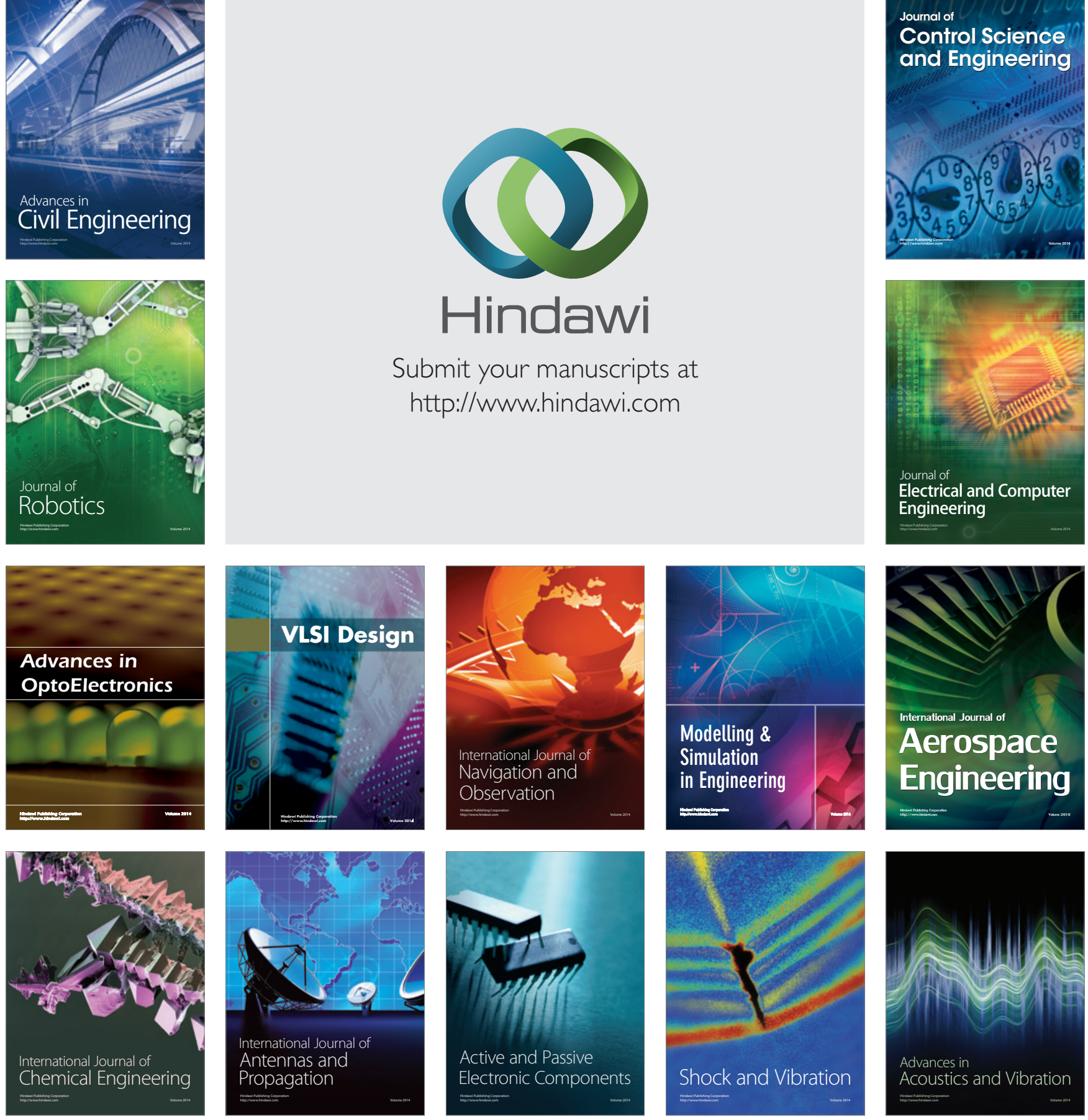